\title{
ANYONS: MINIMAL AND EXTENDED FORMULATIONS
}

\author{
José L. Cortés*and Mikhail S. Plyushchay ${ }^{\dagger}$ \\ Departamento de Física Teórica, Facultad de Ciencias \\ Universidad de Zaragoza, 50009 Zaragoza, Spain \\ To appear in Mod. Phys. Lett. A
}

\begin{abstract}
The minimal (reduced) and extended canonical formulations for $(2+1)$ dimensional fractional spin particles are considered. We investigate the relationship between them, clearing up the meaning of the coordinates for such particles, and analyse the related question of correlation between spin and momentum. The classical lagrangian corresponding to the extended canonical formulation is constructed, and its gauge symmetries are identified.
\end{abstract}

*e-mail: cortes@dedalo.unizar.es

${ }^{\dagger}$ On leave of absence from the Institute for High Energy Physics, Protvino, Russia; e-mail: mikhail@dedalo.unizar.es 


\section{Introduction}

Besides the generally accepted approach to the description of anyons [1] involving the statistical Chern-Simons U(1) gauge field [2]-[4], there is, in principle, another possibility to describe such $(2+1)$-dimensional fractional spin particles. It consists in using multi-valued representations of the Lorentz group $S O(2,1)[5]-[7]$, or infinite-dimensional unitary representations of its universal covering group $\overline{S L(2, R)}[7]-[12$. The latter case is based on the use of some internal spin vector variables at the classical level which lead to the above mentioned representations after quantization. Moreover, one can try to formulate the theory proceeding from the classical description of relativistic particles with arbitrary spin in terms of noncommuting (in the sense of brackets) coordinates, not using additional spin variables at all [13, 8, 14.

The present paper is devoted to the investigation of the relationship between the latter minimal classical formulation and the extended formulation corresponding to the approach that uses infinite-dimensional unitary representations of $\overline{S L(2, R)}$. In particular, we shall investigate the correlation between spin and momentum for a particle in $2+1$ dimensions. This question is important for anyon physics: recently the property of parallelness of spin and momentum has been used in Ref. [15] as a guiding principle for the introduction of the electromagnetic interaction of fractional spin particles within the framework of the minimal formulation

The paper is organized as follows. In sect. 2 proceeding from the general reasonings, we introduce the minimal (reduced) and extended canonical formulations for a $(2+1)$ dimensional particle with arbitrary spin and then investigate in detail the first case. Here we consider the question of correlation between spin and momentum and discuss the quantization of the minimal formulation. Sect. 3 is devoted to the extended formulation. First we trace its relationship to the minimal formulation and then discuss the lagrangian approach corresponding to it, identifying the local gauge symmetries of the classical action. Section 4 is devoted to concluding remarks.

\section{Minimal formulation}

As it is well known [3, [4], in $2+1$ dimensions the spin is a pseudoscalar variable. So, at the classical level, within the canonical approach a particle with fixed mass $m>0$ and spin $s \neq 0$ has the same number of degrees of freedom as a scalar particle, and we can describe it in the following way. Introduce the coordinate $x_{\mu}$ and momentum $p_{\mu}$ variables, considering $p_{\mu}, \mu=0,1,2$, as the components of the energy-momentum vector being the generator of space-time translations. Therefore, we postulate the classical brackets:

$$
\begin{aligned}
& \left\{p_{\mu}, p_{\nu}\right\}=0 \\
& \left\{x_{\mu}, p_{\nu}\right\}=\eta_{\mu \nu}
\end{aligned}
$$

where $\eta_{\mu \nu}=\operatorname{diag}(-,+,+)$. Then the total angular momentum for a spinning particle can be taken in the form generalizing that for the scalar particle:

$$
\mathcal{J}_{\mu}=-\epsilon_{\mu \nu \lambda} x^{\nu} p^{\lambda}+J_{\mu}
$$

\footnotetext{
${ }^{1}$ It has been shown [15] that the value of the gyromagnetic ratio for charged anyons, previously obtained in a standard approach involving the statistical Chern-Simons gauge field [16], is essentially a reflection of the parallelness of spin and momentum.
} 
Here $\mathcal{J}_{\mu}$ is the vector dual to the total angular momentum tensor $\mathcal{J}^{\nu \lambda}, \mathcal{J}_{\mu}=-\frac{1}{2} \epsilon_{\mu \nu \lambda} \mathcal{J}^{\nu \lambda}$, and $\epsilon_{\mu \nu \lambda}$ is a totally antisymmetric tensor, $\epsilon^{012}=1$. $\mathcal{J}_{\mu}$ together with $p_{\mu}$ are the generators of the classical Poincaré group, and so, brackets (2.1) should be supplemented with the brackets

$$
\begin{aligned}
\left\{\mathcal{J}_{\mu}, \mathcal{J}_{\nu}\right\} & =-\epsilon_{\mu \nu \lambda} \mathcal{J}^{\lambda} \\
\left\{\mathcal{J}_{\mu}, p_{\nu}\right\} & =-\epsilon_{\mu \nu \lambda} p^{\lambda}
\end{aligned}
$$

The condition that the particle has a fixed mass can be taken into account by means of the constraint

$$
p^{2}+m^{2} \approx 0
$$

fixing the value of the classical analog of the corresponding Casimir operator of the quanum mechanical Poincaré group $\overline{I S O(2,1)}$. The second term $J_{\mu}$ in (2.3) takes into account the nontrivial spin of the particle $s \neq 0$, and one can consider two different cases. In the first case we can suppose that $J_{\mu}$ depends on some $2 n$ internal phase space variables, independent on $x_{\mu}$ and $p_{\mu}$, i.e. $\left\{J_{\mu}, x_{\nu}\right\}=\left\{J_{\mu}, p_{\nu}\right\}=0$, and that the coordinates of the particle $x_{\mu}$ are commuting in the sense of the brackets,

$$
\left\{x_{\mu}, x_{\nu}\right\}=0
$$

Then $J_{\mu}, \mu=0,1,2$, have to form the algebra of Lorentz generators

$$
\left\{J_{\mu}, J_{\nu}\right\}=-\epsilon_{\mu \nu \lambda} J^{\lambda}
$$

and the constraint

$$
p J-s m \approx 0
$$

will fix the value of particle spin in correspondence with relations (2.3), (2.6) and the definition

$$
S=\frac{p^{\mu} \mathcal{J}_{\mu}}{\sqrt{-p^{2}}}
$$

for the classical analog of the spin Casimir operator of the Poincaré group. Since the first class constraint (2.9) cancels one phase space degree of freedom (2 variables), we have to supplement it with the corresponding number of first and (or) second class constraints to cancel the remaining $2(n-1)$ internal phase space variables. We shall consider in detail an example with additional internal phase space variables in the next section and now turn to the minimal formulation involving only phase space variables $x_{\mu}$ and $p_{\mu}$. In this case due to eqs. (2.3) and (2.10), $J_{\mu}$ must have the form

$$
J_{\mu}=-s e_{\mu}^{(0)}+J^{(i)} e_{\mu}^{(i)},
$$

where we have introduced the triad $e_{\mu}^{(\alpha)}=e_{\mu}^{(\alpha)}(p), \alpha=0,1,2, e_{\mu}^{(0)}=p_{\mu} / \sqrt{-p^{2}}$,

$$
e_{\mu}^{(\alpha)} \eta_{\alpha \beta} e_{\nu}^{(\beta)}=\eta_{\mu \nu}, \quad e_{\mu}^{(\alpha)} \eta^{\mu \nu} e_{\nu}^{(\beta)}=\eta^{\alpha \beta}, \quad \epsilon^{\mu \nu \lambda} e_{\mu}^{(0)} e_{\nu}^{(1)} e_{\lambda}^{(2)}=1
$$

Due to eqs. (2.2) and (2.5), $J^{(i)}, i=1,2$, can depend only on $p_{\mu}$. Therefore, the vector $\mathcal{J}_{\mu}$ can be presented in the equivalent form:

$$
\mathcal{J}_{\mu}=-\epsilon_{\mu \nu \lambda} \tilde{x}^{\nu} p^{\lambda}-s e_{\mu}^{(0)}
$$


where

$$
\tilde{x}_{\mu}=x_{\mu}-\epsilon^{0 i j} \frac{J^{(i)}}{\sqrt{-p^{2}}} e_{\mu}^{(j)}=x_{\mu}+\frac{1}{p^{2}} \epsilon_{\mu \nu \lambda} p^{\nu} J^{\lambda} .
$$

The elongated variables $\tilde{x}_{\mu}$ have the same brackets with $p_{\mu}$ as $x_{\mu}$, whereas the form of brackets (2.4) partially fixes the form of the brackets between different components $\tilde{x}_{\mu}$ :

$$
\left\{\tilde{x}_{\mu}, \tilde{x}_{\nu}\right\}=\epsilon_{\mu \nu \lambda} R^{\lambda}, \quad R_{\mu}=-s \frac{p_{\mu}}{\left(-p^{2}\right)^{3 / 2}}+R^{(i)} e_{\mu}^{(i)}
$$

where $R^{(i)}$ are arbitrary functions of $p_{\mu}$. So, for a particle with fixed mass $m$ and spin $s$, we have the classical Poincare algebra (2.1), (2.4), (2.5), but the brackets $\left\{x_{\mu}, x_{\nu}\right\}$ for the initial variables as well as those for elongated coordinates (2.15) are still unfixed.

Let us introduce now the additional requirement for the variables $\tilde{x}_{\mu}, \mu=0,1,2$, to be the three components of a vector, i.e.

$$
\left\{\mathcal{J}_{\mu}, \tilde{x}_{\nu}\right\}=-\epsilon_{\mu \nu \lambda} \tilde{x}^{\lambda}
$$

This requirement leads to the prescription $R^{(i)}=0$, and completely fixes brackets (2.15):

$$
\left\{\tilde{x}_{\mu}, \tilde{x}_{\nu}\right\}=-s \epsilon_{\mu \nu \lambda} \frac{p^{\lambda}}{\left(-p^{2}\right)^{3 / 2}} .
$$

Therefore, in order to have the system completely defined, we must interprete the vector components $\tilde{x}_{\mu}, \mu=0,1,2$, as the coordinates of the particle with spin $s$, and in this case the form of the angular momentum vector (2.13) will be completely fixed. Then the initial coordinates $x_{\mu}$ could be considered as some auxiliary variables, and after taking into account eqs. (2.14) and (2.16), we find that they do not form a Lorentz vector. We shall discuss this noncovariance in the next section.

Obviuosly, we could postulate the covariant brackets of the form (2.17) for the initial variables $x_{\mu}$ from the very beginning, and work in terms of only such covariant variables. Then the addition $J_{\mu}$ would be fixed in the same covariant form as in eq. (2.13), i.e. we would have $J^{(i)}=0$. But, as we shall see below, the manifest covariance of the minimal formulation will be inevitably lost under transition to the quantum theory even if we work with the vector variables $\tilde{x}_{\mu}$. Therefore, the construction of the minimal formulation, which includes $x_{\mu}$ as the original noncovariant variables, is also useful for a general discussion of the formulation. Moreover, as we shall see, such a construction will help us to understand its relationship to the formulations based on the addition of internal phase space variables.

Before the discussion of quantization, let us stress that only after fixing the notion of the particle coordinates as the components of a Lorentz vector, the additional term in the total angular momentum vector (2.13) will be parallell to the energy-momentum vector $p^{\mu}$. This second term has nontrivial brackets with the first one looking formally as the orbital angular momentum, and, moreover, the components of neither first nor second terms form themselves the algebra of Lorentz generators.

Concluding this section, let us demonstrate that the manifest covariance of the formulation is lost under the transition to the quantum theory. Indeed, the problem of quantization is reduced to the problem of constructing the quantum analogs of the variables $\tilde{x}_{\mu}$ having nontrivial brackets (2.17). Such operators can be constructed in the 
following way. Let us introduce the variables $q_{\mu}$,

$$
\begin{gathered}
\tilde{x}_{\mu}=q_{\mu}+A_{\mu}, \\
\left\{q_{\mu}, q_{\nu}\right\}=0, \quad\left\{q_{\mu}, p_{\nu}\right\}=\eta_{\mu \nu}
\end{gathered}
$$

by defining the "gauge potential" $A_{\mu}=A_{\mu}(p)$ with the help of the monopole-like relation [13, 8]:

$$
\partial_{\mu} A_{\nu}-\partial_{\nu} A_{\mu}=-s \epsilon_{\mu \nu \lambda} \frac{p^{\lambda}}{\left(-p^{2}\right)^{3 / 2}} .
$$

This relation defines the "gauge potential" up to a "gauge transformation", $A_{\mu} \rightarrow A_{\mu}^{\prime}=$ $A_{\mu}+\partial_{\mu} f$, and we can choose it in the form:

$$
A_{\mu}=-s \epsilon_{\mu \nu \lambda} \frac{p^{\nu} \xi^{\lambda}}{p^{2}+\sqrt{-p^{2}}(p \xi)},
$$

where $\xi^{\mu}$ is an arbitrary fixed timelike unit vector, $\xi^{2}=-1$ (that can be taken, e.g., as $\left.\xi^{\mu}=(1,0,0)\right)$. Whence we immediately conclude that due to the noncovariant form of the "gauge potential" (2.20) and the vector character of $\tilde{x}_{\mu}$, the variables $q_{\mu}$ do not form a Lorentz vector having complicated transformation properties under Lorentz boosts, and, therefore, we loose here the manifest covariance of the formulation. But these variables give the possibility to realize the operators corresponding to the classical variables $\tilde{x}_{\mu}$ in the obvious way using the quantum analog of eq. (2.18).

So, we see that the minimal formulation loses its manifest covariance under transition to the quantum theory.

\section{Extended formulation}

Let us turn now to the extended formulation with phase space variables $x_{\mu}, p_{\mu}, J_{\mu}$, and brackets (2.1), (2.2), (2.7), (2.8). The total angular momentum vector is given by (2.3) and the phase space variables are constrained by the mass and spin conditions (2.6), (2.9). First of all, we note that the scalar $J^{2}$ lies in the center of algebra (2.8), $\left\{J^{2}, J_{\mu}\right\}=0$, and, therefore, it can be fixed:

$$
J^{2}=C,
$$

where $C$ is some real constant. Therefore, due to eq. (3.1), the number of independent internal phase space variables will be equal to 2 . So, we shall have the minimally extended formulation.2 Then for $C=-\alpha^{2}, \alpha>0$, eq. (3.1) sets two disconnected sheets of the hyperboloid:

$$
J_{0}=\varepsilon \sqrt{\alpha^{2}+J_{i}^{2}}, \quad \varepsilon= \pm, \quad i=1,2,
$$

whereas in the case $C=\beta^{2} \geq 0$ it defines a one-sheet hyperboloid (degenerating into the cone at $\beta=0)$. Note here, that in correspondence with this property, the quantization of the classical subsystem, defined by relations (2.8) and (3.1), results in the infinitedimensional unitary irreducible representations of the group $\overline{S L(2, R)}$ either of the halfbounded discrete type series $D_{\alpha}^{ \pm}$(for $C=-\alpha^{2}<0$ ) or of the continuous series (for

\footnotetext{
${ }^{2}$ The most general case for the extended formulation and its relation to the minimal formulation will be considered elsewhere 17 .
} 
$\left.C=\beta^{2} \geq 0\right)$, where the operator $\hat{J}_{0}$ takes correspondingly the eigenvalues $j_{0}= \pm(\alpha+n)$, $n=0,1,2, \ldots$, or $j_{0}=\theta+n, \theta \in[0,1), n=0, \pm 1, \pm 2, \ldots$ (see Ref. 18 for the details).

Now, let us reveal the relationship of the extended formulation to the minimal one. To this end we use the complete triad (2.12) to represent the vector $J_{\mu}$ in the form $J_{\mu}=e_{\mu}^{(\alpha)} \eta_{\alpha \beta} J^{(\beta)}$. The quantities $J^{(\alpha)}=e_{\mu}^{(\alpha)} J^{\mu}$ satisfy the same algebra as the initial variables $J^{\mu}:\left\{J^{(\alpha)}, J^{(\beta)}\right\}=-\epsilon^{\alpha \beta \gamma} J_{(\gamma)}$, and the spin condition (2.9) can be presented in the equivalent form:

$$
J^{(0)}-s \approx 0
$$

Taking into account condition (3.1), we find that the constraint (3.3) singles out the circle $S^{1}, J^{(0)}=s, J^{(i)} J^{(i)}=s^{2}+C$, as a physical subspace, i.e. classical conditions (3.1) and (3.3) are simultaneuosly consistent for all the values of spin $s$ when $C \geq 0$, but they are consistent only for $s^{2} \geq \alpha^{2}$ for the case $C=-\alpha^{2}<0$. The circle shrinks into just a one point $J^{(0)}=s, J^{(i)}=0$ only when $-J^{2}=\alpha^{2}=s^{2}$. Only in this special case the spin vector $J_{\mu}$ is parallel (in a weak sense) to the momentum vector $p_{\mu}$.

The vector $p^{\mu}$ and the elongated coordinates $\tilde{x}_{\mu}$ constructed from $x_{\mu}$ according to the prescription of the same form as in eq. (2.14), have zero brackets with constraint (3.3) and, therefore, they (together with $J^{(0)}$ ) are the only gauge-invariant variables with respect to the gauge transformations generated by this constraint. The transformations are reduced simply to the rotation for the variables $J^{(i)}, i=1,2$, and the gauge orbit here is just the same circle $S^{1}$ shrinking into a point in the above special case.

The three $J_{(\alpha)}$ can be parametrized in the following general form:

$$
J_{(\alpha)}=\left(J_{(0)}, J_{(i)}\right)=\left(J_{(0)}, \sqrt{J_{(0)}^{2}+C} \cdot n_{i}\right), \quad n_{i}=(\cos \phi, \sin \phi),
$$

where $0 \leq \phi<2 \pi$ and $-\infty<J_{(0)}<\infty$ in the case $C \geq 0$, whereas $J_{(0)}$ can take values in the region $[\alpha,+\infty)$ or $(-\infty,-\alpha]$ when $J^{2}=-\alpha^{2}<0$. Then, proceeding from brackets for the variables $J_{(\alpha)}$, we find that the brackets for the independent variables $J_{(0)}$ and $\phi$ have the form

$$
\left\{\phi, J_{(0)}\right\}=1 .
$$

Now we can reduce the system to the surface defined by the spin constraint (3.3). The reduction consists here in choosing some point on the gauge orbit $S^{1}$. This can be done, for example, with the help of the (local) gauge condition:

$$
\phi-\phi_{0} \approx 0
$$

where $\phi_{0}$ is some fixed point, $\phi_{0} \in S^{1}$. Note, that the reduction can also be applied to the special case $-J^{2}=\alpha^{2}=s^{2}$ if it is considered as a limit, e.g., $J^{2}=-\alpha^{2}, s^{2}=\alpha^{2}+\epsilon^{2}$, $\epsilon \rightarrow 0$. After calculating the Dirac brackets with the help of second class constraints (3.3) and (3.6), the "spin vector" $J^{\mu}$ is completely fixed:

$$
J_{\mu}=-s e_{\mu}^{(0)}+\gamma_{i} e_{\mu}^{(i)},
$$

where the constants $\gamma_{i}$ are given in terms of the angle $\phi_{0}$ via the parametrization (3.4). The derivation of Dirac brackets is simplified if one uses the elongated gauge invariant variables (2.14) whose Dirac brackets $\left\{\tilde{x}_{\mu}, \tilde{x}_{\nu}\right\}^{*}$ coincide with the initial brackets. The brackets $\left\{\tilde{x}_{\mu}, \tilde{x}_{\nu}\right\}$ have here the same form (2.17) as for the variables $\tilde{x}_{\mu}$ in the minimal formulation. The result for the Dirac brackets of the coordinates $x_{\mu}$ is:

$$
\left\{\mathcal{J}_{\mu}, x_{\nu}\right\}^{*}=-\epsilon_{\mu \nu \lambda} x^{\lambda}+\gamma_{i} e_{\nu}^{(i)}\left(-\frac{e_{\mu}^{(0)}}{\sqrt{-p^{2}}}+e_{\mu}^{(j)} \partial^{\sigma} e_{\sigma}^{(j)}\right)
$$




$$
\left\{x_{\mu}, x_{\nu}\right\}^{*}=\epsilon_{\mu \nu \lambda} \frac{e^{(0) \lambda}}{\sqrt{-p^{2}}} \partial^{\sigma}\left(-\frac{1}{2} s e_{\sigma}^{(0)}+\gamma_{i} e_{\sigma}^{(i)}\right) .
$$

Therefore, as follows from eqs. (3.8) and (3.7), in the general case neither coordinates $x^{\mu}$ nor "spin" $J^{\mu}$ are any more Lorentz vectors after reduction and, besides, $J^{\mu}$ is not parallel to the energy-momentum vector $p^{\mu}$. Moreover, brackets (3.9) generally have a noncovariant form. All these noncovariant properties appear because the variables $x_{\mu}$ and $J_{\mu}$ are gauge nonivariant and they feel the noncovariance of the gauge condition (3.6). Only in the case $\gamma_{i}=0$, (i.e. only when $-J^{2}=\alpha^{2}=s^{2}$ ) this noncovariance in the reduced system dissapears, and then $x_{\mu}$ coincide with the gauge invariant coordinates $\tilde{x}_{\mu}$ and brackets (3.9) turn into the covariant brackets (2.17). Thus, from the point of view of the reduction of the system the case $-J^{2}=\alpha^{2}=s^{2}$ is again a special one: only in this case $x_{\mu}$ remains a Lorentz vector, and the brackets between different components have a covariant (but nonzero) form. It is just only in this case that the gauge noninvariant spin vector $J^{\mu}$ becomes parallel to the momentum vector $p^{\mu}$ after reduction.

Concluding the discusion of the canonical approach, we note that the Dirac brackets (3.8), (3.9) for the variables $x_{\mu}$ coincide with the brackets for $x_{\mu}$ in the minimal formulation for a choice of the addition (2.11) in the form (3.7) (i.e. for $J^{(i)}=\gamma^{i}=$ const). So, the minimal formulation presented in terms of the variables $x_{\mu}$ is nothing else than the reduced system of the extended formulation, and the elongated covariant coordinates $\tilde{x}_{\mu}$ given by eq. (2.14) are the gauge invariant coordinates of the extended formulation.

Now, let us consider the lagrangian approach corresponding to the described extended canonical system.

The brackets (2.8) for the internal variables $J_{\mu}$ can be derived from a kinetic lagrangian

$$
L_{k i n}=-\frac{J \xi}{J^{2}+(J \xi)^{2}} \epsilon_{\mu \nu \lambda} \xi^{\mu} J^{\nu} \dot{J}^{\lambda}
$$

with arbitrary fixed unit timelike vector $\xi^{\mu}, \xi^{2}=-1$. The simplest way to be convinced that it is so consists in checking the fact that under a Lorentz transformation of $J_{\mu}$, the kinetic term (3.10) is changed by a total derivative, and, therefore, it corresponds to a Lorentz invariant term in the action. Then, choosing $\xi^{\mu}=(1,0,0)$, and parametrizing the variables $J_{\mu}$ as in eq. (3.4), $J_{\mu}=J_{\mu}\left(J_{0}, \varphi\right)$, one gets $L_{k i n}=J_{0} \cdot \dot{\varphi}$. From here we find that the brackets for independent variables $J_{0}$ and $\varphi$ have the form (3.5) (with the substitution $J_{(0)}$ for $J_{0}$ and $\phi$ for $\varphi$ ), and, therefore, the Lagrangian (3.10) indeed leads to the brackets (2.8).

We shall obtain the total lagrangian of the extended formulation by adding to the kinetic term a lagrangian depending on $\dot{x}_{\mu}$ and $J_{\mu}$ (and on auxiliary Lagrange multipliers), which will lead to the constraints (2.6) and (2.9), and, so, describe a system with fixed mass and spin. Such an addition can be constructed proceeding from the well known lagrangian approach for a relativistic spin- $1 / 2$ particle. For example, one can take the pseudoclassical lagrangian for the Dirac particle proposed in Ref. [19] with corresponding substitution of odd variables by even ones and one gets

$$
L=\frac{1}{2 e}\left(\dot{x}_{\mu}-v J_{\mu}\right)^{2}-\frac{1}{2} e m^{2}+s m v+L_{k i n} .
$$

Lagrangian (3.11), with $e$ and $v$ being the Lagrange multipliers, leads to the mass and spin conditions (2.6) and (2.9) as secondary constraints, and, therefore, it is the lagrangian corresponding to the extended canonical system discussed above. 
The action $A=\int L d \tau$ is invariant with respect to the reparametrizations: $\delta x_{\mu}=\gamma \dot{x}_{\mu}$, $\delta J_{\mu}=\gamma \dot{J}_{\mu}, \delta e=(\gamma e) ; \delta v=(\gamma v) ; \delta L=\frac{d}{d \tau}(\gamma L), \gamma=\gamma(\tau)$, whose generator is the mass shell constraint, and, moreover, it is invariant with respect to the transformations generated by the spin constraint (2.9):

$$
\begin{gathered}
\delta e=0, \quad \delta v=\dot{\rho}, \quad \delta x_{\mu}=\rho J_{\mu}, \quad \delta J_{\mu}=-\rho e^{-1} \epsilon_{\mu \nu \lambda} \dot{x}^{\nu} J^{\lambda} \\
\delta L=\frac{d}{d \tau}\left(\rho\left(s m+e^{-1} \dot{x} J-J^{2} e^{-1}(\dot{x} J+(\dot{x} \xi)(\xi J)) \cdot\left(J^{2}+(J \xi)^{2}\right)^{-1}\right)\right),
\end{gathered}
$$

where $\rho=\rho(\tau)$. The conservation of these lagrangian symmetries can be used as a general guiding principle for the extension of the system to the case of its interaction with gauge fields, e.g. with a $\mathrm{U}(1)$ gauge field in the simplest case [17].

Let us consider now the Lagrange equations of motion for $e$ and $v$ following from (3.11):

$$
\left(\dot{x}_{\mu}-v J_{\mu}\right)^{2}+e^{2} m^{2}=0, \quad \dot{x} J-v J^{2}-s m e=0 .
$$

From the second equation we get the equality $e=s^{-1} m^{-1}\left(\dot{x} J-v J^{2}\right)$. Putting it into the first equation, we arrive at the relation $\dot{x}^{2}+s^{-2}(\dot{x} J)^{2}=-v\left(1+s^{-2} J^{2}\right) \cdot\left(v J^{2}-2 \dot{x} J\right)$. From here we conclude that iff

$$
-J^{2}=\alpha^{2}=s^{2},
$$

there is the Lagrange constraint:

$$
\dot{x}^{2}+s^{-2}(\dot{x} J)^{2}=\dot{x}^{2}-(\dot{x} J)^{2} \cdot\left(J^{2}\right)^{-1}=0,
$$

which means that the particle velocity vector $\dot{x}_{\mu}$ is paralell to the spin vector $J_{\mu}$.

To conclude this section, let us rewrite lagrangian (3.11) in a form revealing the speciality of the case (3.13) in a more explicite way. To this end, we find the multiplier $v$ from the second equation (3.12) assuming that $J^{2} \neq 0, v=\left(J^{2}\right)^{-1}(\dot{x} J-s m e)$, and put it into lagrangian (3.11). Then we get the following form for the total lagrangian:

$$
L=\frac{1}{2 e}\left(\dot{x}^{2}-\left(J^{2}\right)^{-1}(\dot{x} J)^{2}\right)+s m\left(J^{2}\right)^{-1}(\dot{x} J)-\frac{1}{2} e m^{2}\left(1+s^{2}\left(J^{2}\right)^{-1}\right)+L_{k i n} .
$$

The term linear in $e$ dissapears from (3.15) only when eq. (3.13) takes place, and as a consequence, the variation of the corresponding action through $e$ gives the Lagrange constraint (3.14). The spin constraint (2.9) appears as the primary constraint in this case, whereas the mass-shell constraint (2.6) is a secondary one.

\section{Concluding remarks}

Proceeding from the classical canonical consideration of the relativistic fractional spin particles, which does not involve Chern-Simons U(1) gauge field constructions, we have shown that there are (at least) two different approaches to the description of anyons with or without an explicit spin degree of freedom. We have investigated the both cases, called here the minimal and extended formulations, revealing their mutual relationship and constructing the lagrangian approach corresponding to the latter formulation.

It has been demonstrated that within the minimal approach, the spin term in the total angular momentum vector becomes parallel to the energy-momentum vector of the 
particle with nonzero arbitrary spin only after fixing the notion of the particle coordinates as those forming a Lorentz vector. This fact is nontrivial. We have shown that the canonical system for the minimal formulation corresponds to the system appearing after reduction of the extended canonical system to the surface defined by the spin constraint (2.9). In such reduced system the initial coordinates of the particle do not remain the components of a Lorentz vector, but the gauge-invariant elongated coordinates form a Lorentz vector and their Dirac brackets coincide with the nontrivial brackets (2.17) for covariant coordinates in the minimal formulation.

The case $-J^{2}=\alpha^{2}=s^{2}$ for the particle with spin $s$, being contained in the extended formulation and corresponding to the choice of the discrete type series of representations $D_{\alpha}^{ \pm}$at the quantum level [7, 18], is a special one. Only in this case internal spin vector $J^{\mu}$ is parallel to the energy-momentum vector $p^{\mu}$ (in a weak sense, or in a strong sense after reduction to the spin constraint surface (2.9)), and the initial coordinates of the particle coincide here with the above mentioned gauge-invariant coordinates. Within the corresponding lagrangian formulation this case is also a special one: only in this case there is a lagrangian constraint in the system that prescribes the velocity of the particle to be parallel to the time-like spin vector $J_{\mu}$.

We have demonstrated that the manifest covariance of the minimal formulation is inevitably lost after transition to the quantum theory. This is due to the monopolelike equation (2.19) for the "gauge potential" which appears in the construction of the "localizable" commuting coordinates $q_{\mu}$. Such coordinates $q_{\mu}$, having complicated transformation properties under Lorentz boosts, are analogs of the Newton-Wigner coordinates [20]. Though similar noncovariance takes place also for the extended formulation at the lagrangian level due to the presence of nondynamical arbitrary fixed timelike unit vector $\xi_{\mu}$ in the kinetic term (3.10), the final hamiltonian formulation (together with the corresponding quantum theory [9, 7]) is, nevetherless, manifestly covariant.

This work was partially supported by MEC-DGICYT (Spain). 


\section{References}

[1] J.M. Leinaas and J. Myrheim, Nouvo Cimento B37, 1 (1977);

G.A. Goldin, R. Menikoff and D.H. Sharp, J. Math. Phys. 22, 1664 (1981);

F. Wilczek, Phys. Rev. Lett. 48, 1144 (1982); 49, 957 (1982).

[2] G.W. Semenoff, Phys. Rev. Lett. 61, 517 (1988).

[3] P. Gerbert, Intern. J. Mod. Phys. A6, 173 (1991);

S. Forte, Rev. Mod. Phys. 64, 193 (1992);

K. Lechner and R. Iengo, Phys. Rep. 213, 179 (1992).

[4] F. Wilczek, Fractional Statistics and Anyon Superconductivity, (World Scientific, Singapore, 1990).

[5] M.S. Plyushchay, Phys. Lett. B248, 107 (1990).

[6] S. Forte and T. Jolicoeur, Nucl. Phys. B350, 589 (1991).

[7] M.S. Plyushchay, Intern. J. Mod. Phys. A7, 7045 (1992).

[8] R. Jackiw and V.P. Nair, Phys. Rev. D43, 1933 (1991).

[9] M.S. Plyushchay, Phys. Lett. B262, 71 (1991).

[10] D.P. Sorokin and D.V. Volkov, Nucl. Phys. B409, 547 (1993).

[11] M.S. Plyushchay, Phys. Lett. B320, 91 (1993).

[12] J.L. Cortés and M.S. Plyushchay, J. Math. Phys. 35, 6049 (1994).

[13] J.F. Schonfeld, Nucl. Phys. B185, 117 (1981).

[14] R. Jackiw and V.P. Nair, hep-th/9403010 preprint.

[15] C. Chou, V.P. Nair and A.P. Polychronakos, Phys. Lett. B304, 105 (1993).

[16] I.I. Kogan, Phys. Lett. B262, 83 (1991);

J.L. Cortés, J.Gamboa and L. Velázquez, Phys. Lett. B286, 105 (1992); Nucl. Phys. B392, 645 (1993).

[17] J.L. Cortés and M.S. Plyushchay, in preparation.

[18] M.S. Plyushchay, J. Math. Phys. 34, 3954 (1993).

[19] J.L. Cortés, M.S. Plyushchay and L. Velázquez, Phys. Lett. B306, 34 (1993).

[20] T.D. Newton and E.P. Wigner, Rev. Mod. Phys. 21, 400 (1949). 